\title{
Crecimiento de Pennisetum purpureum cv. Taiwán con diferentes fuentes de fertilización en pastoreo con bovinos
}

\section{Growth of Pennisetum purpureum cv. Taiwan with different sources of fertilization in bovine grazing}

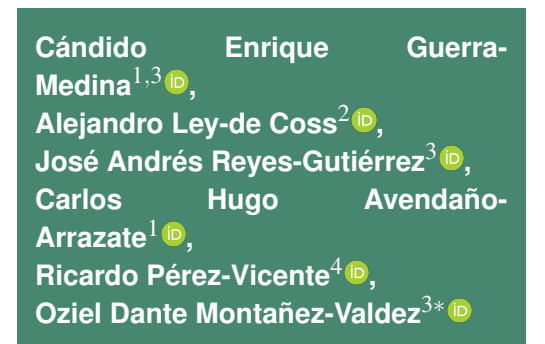

${ }^{1}$ Instituto Nacional de Investigaciones Forestales Agrícolas y Pecuarias (INIFAP), Campo Experimental, Rosario Izapa, Tuxtla Chico, Chiapas, México.

${ }^{2}$ Facultad de Ciencias Agronómicas Campus V, UNACH, Villaflores, Chiapas, México.

${ }^{3}$ Grupo de Investigación en Nutrición Animal, Centro Universitario del Sur, Universidad de Guadalajara, Ave. Enrique Arreola Silva 883, Ciudad Guzmán, Jalisco, México.

${ }^{4}$ Centro Universitario de la Costa Sur, Universidad de Guadalajara, Autlán de Navarro, Jalisco, México.

*Autor de correspondencia: montanez77@hotmail.com

\section{Artículo científico}

Recibido: 08 de febrero 2021

Aceptado: 11 de septiembre 2021

Como citar: Guerra-Medina CE, Ley-de Coss A, Reyes-Gutiérrez $\mathrm{JA}$, Avendaño-Arrazate $\mathrm{CH}$, PérezVicente R, Montañez-Valdez OD (2021) Crecimiento de Pennisetum purpureum cv. Taiwán con diferentes fuentes de fertilización en pastoreo con bovinos. Ecosistemas y Recursos Agropecuarios Núm. Esp. II: e2859. DOI: 10.19136/era.a8nll.2859
RESUMEN. El objetivo fue evaluar el efecto de dos fuentes de fertilización sobre la composición química, características de crecimiento y persistencia del pasto Taiwán bajo pastoreo intensivo. Los tratamientos fueron: sin fertilización (SF), fertilizante húmico $(\mathrm{FH})$, fertilizante granulado $(\mathrm{FG})$. También se evaluaron dos alturas de corte después del pastoreo; sin corte de uniformidad (SC); altura de corte a 30 $\mathrm{cm}$ (C30), altura de corte a $50 \mathrm{~cm}$ (C50). Los tratamientos se distribuyeron en un diseño bloques al azar, se aplicó un análisis de varianza y pruebas de medias. Las variables evaluadas fueron: composición química, altura de planta $(A P)$, largo de hoja (LH), ancho de hoja $(A H)$, peso seco de planta (PP), peso de tallo (PT), peso de hoja $(\mathrm{PH})$, porcentaje de tallo, porcentaje de hoja, número de hijuelos $(\mathrm{NH})$ y número de macollos vivos (MV). El contenido de proteína cruda aumentó de 7.99 a 9.31 y $11.87 \%$ en los tratamientos SF, FH y FG de manera respectiva; no hubo efecto de la fuente de fertilización en AP, AH, PP, PT, PH, porcentaje de tallo, porcentaje de hoja, $\mathrm{NH}$ y $\mathrm{MV}(\mathrm{P}>0.05)$; mientras que el largo de hoja fue menor ( $\mathrm{P}$ $<0.05$ ) en $\mathrm{FH}$. El corte de uniformidad después del pastoreo a 30 y $50 \mathrm{~cm}$, aumentó $\mathrm{NH}(\mathrm{P}<0.05)$. La aplicación de fertilizante húmico y granulado al pasto Taiwán, aumentó el contenido de proteína; el corte de uniformidad a 30 y $50 \mathrm{~cm}$, estimuló el desarrollo de mayor número de hijuelos, lo que promueve la persistencia de la pradera.

Palabras clave: Forraje, manejo, nutrientes, pastoreo, trópico.

ABSTRACT. The objective of the study was to evaluate the effect of two sources of fertilization on the chemical composition, growth characteristics and persistence of Taiwan grass under intensive grazing. The treatments were: no fertilization $(\mathrm{SF})$, humic fertilizer (FH), granulated fertilizer (FG). Two cutting heights were also evaluated after grazing; no uniformity cut (SC); cutting height at $30 \mathrm{~cm}$ (C30), cutting height at $50 \mathrm{~cm}(\mathrm{C50})$. The treatments were distributed in a randomized block design, an analysis of variance and tests of means were applied. The variables evaluated were: chemical composition, plant height (AP), leaf length (LH), leaf width $(\mathrm{AH})$, plant dry weight $(\mathrm{PP})$, stem weight $(\mathrm{PT})$, leaf weight $(\mathrm{PH})$, stem percentage, leaf percentage, number of suckers $(\mathrm{NH})$ and number of live tillers $(\mathrm{MV})$. The crude protein content increased from 7.99 to 9.31 and $11.87 \%$ in the SF, FH and FG treatments respectively; there was no effect of the fertilization source on $A P, A H$, $\mathrm{PP}, \mathrm{PT}, \mathrm{PH}$, stem percentage, leaf percentage, $\mathrm{NH}$ and $\mathrm{MV}(\mathrm{P}>0.05)$; while the leaf length was less $(\mathrm{P}<0.05)$ in $\mathrm{FH}$. The uniformity cut after grazing at 30 and $50 \mathrm{~cm}$, increased $\mathrm{NH}(\mathrm{P}<0.05)$. The application of humic and granulated fertilizer to Taiwan grass increased the protein content; the uniformity cut at 30 and $50 \mathrm{~cm}$, stimulated the development of a greater number of suckers, which promotes the persistence of the meadow.

Key words: Forage, management, nutrients, grazing, tropics. 


\section{INTRODUCCIÓN}

En América Latina $78 \%$ de la ganadería se dedica al sistema bovinos doble propósito en pastoreo extensivo, por lo que, representa una de las principales actividades del sector agropecuario (Perdomo et al. 2017, Molina et al. 2019). Así mismo, en las regiones tropicales de México, predomina la ganadería dedicada a la cría de becerros para producción de carne, leche y doble propósito, con una población de 34820271 cabezas, para ello se dedican 77842256 hectáreas de pastoreo extensivo que representa el $23 \%$ del territorio nacional (SIAP 2019). De esta superficie el $50 \%$ son agostaderos y praderas nativas, $25 \%$ praderas inducidas y el resto praderas introducidas (INEGI 2017). Pero en estas regiones la productividad es baja, uno de los factores limitantes es la alimentación deficiente del ganado por la estacionalidad en la producción forrajera, dependiente de la precipitación, con mayor precipitación en los meses de junio a octubre y la sequía de noviembre a mayo (García 2004). Durante el estiaje el crecimiento de las especies forrajeras es limitado y de menor contenido de nutrientes (May et al. 2011), además de factores como falta de adaptación de la especie forrajera (Araya y Boschinni 2005), y baja eficiencia en la utilización de los recursos (Arboleda et al. 2013). Para incrementar la productividad, se requiere intensificar el proceso de manera sustentable, por lo que es importante estudiar las especies forrajeras que tengan mayor adaptación a las condiciones existentes, mayor productividad y valor nutrimental. El uso de especies forrajeras de corte con mayor productividad, es una práctica creciente en los sistemas de producción ganaderos en el trópico, entre ellas Pennisetum purpureum (López et al. 2020), es una especie originaria de África del Sur, después que fueron introducidas a México tuvieron un proceso de adaptación natural, se encuentran desde 0 a $2200 \mathrm{~m}$ de altitud en diversos suelos, con excepción de aquellos inundables o salinos. Se utiliza en la mayoría de los casos como pasto de corte por su mayor crecimiento, valor nutricional y producción de biomasa por unidad de superficie, resultado de su capacidad fotosintética, eficiente asignación de car- bohidratos hacia las hojas y mayor área foliar específica (Quero y Miranda 2013), con variedades conocidas como Taiwán, Gigante o Elefante, Kin Grass, Merkerón y Napier, además de los cultivares de reciente introducción al país como el OM-22, CT-115 y Maralfalfa (López et al. 2011, Calzada et al. 2014). Por otra parte, el aprovechamiento de luz, $\mathrm{CO}_{2}$, temperatura y agua por las plantas depende de la proporción de hojas, tallos y raíces (Valentine y Mathew 1999). En los pastos Pennisetum spp., el rebrote después del corte depende de las reservas de carbohidratos solubles en el tallo, debido a que la mayor parte del área foliar fue aprovechada, y la cantidad de reservas es mayor en materiales con menos años de establecidos (Calzada et al. 2014). Además de los factores climáticos, el manejo de la especie forrajera como fertilización (Roncallo et al. 2012), altura y edad de corte (Madera et al. 2013), son importantes para obtener mayor rendimiento, calidad nutrimental y asegurar la persistencia de la especie forrajera en el sistema de producción (Ramírez et al. 2010).

Por las características de crecimiento de la especie Pennisetum purpureum, se utiliza como forraje de corte, por lo que la mayoría de los estudios realizados tratan sobre sus características de crecimiento, rendimiento y composición química (González et al. 2011, Nava et al. 2013, Hinojosa et al. 2014). Pero se tienen pocos estudios que evalúen las condiciones de pastoreo intensivo de bovinos para carne, lo que puede afectar la persistencia de la pradera si el manejo no es adecuado. Las implicaciones de utilizar el pasto Taiwán en la producción de carne de bovino en pastoreo intensivo es importante, debido a que puede evitarse el uso de maquinaria para corte y almacenamiento del forraje producido, lo que mejora la rentabilidad y sustentabilidad del sistema. Una variable importante para evaluar la persistencia de esta especie, es el número de hijuelos o nuevas plantas después del corte, debido a que las plantas nuevas son las que permanecen en el siguiente ciclo de pastoreo. Por lo anterior el objetivo de la presente investigación fue evaluar el efecto de aplicar dos fuentes de fertilización y dos alturas de corte sobre las variables de valor nutrimental, crecimiento y persistencia del pasto Taiwán en condiciones de pas- 
toreo intensivo de becerros en engorda.

\section{MATERIALES Y MÉTODOS}

\section{Localización}

El estudio se realizó en los meses de junio de 2018 a octubre de 2019, en una unidad de producción dedicada al sistema bovinos doble propósito, ubicado en el municipio de Tapachula, en el Soconusco, Chiapas, México, ubicada en las coordenadas geográficas: $14^{\circ} 91^{\prime} 36^{\prime \prime}$ LN, $92^{\circ} 32^{\prime} 55^{\prime \prime}$ LO, a $160 \mathrm{~m}$ de altitud. El clima es cálido húmedo con lluvias abundantes en verano, la precipitación pluvial en el periodo de noviembre a abril oscila entre 75 y $800 \mathrm{~mm}$, de mayo a octubre entre 1200 y $3000 \mathrm{~mm}$; y la temperatura oscila entre los 18 y $34.5^{\circ} \mathrm{C}$ (INEGI 2017).

\section{Establecimiento de la pradera}

El material vegetal de pasto Taiwán se obtuvo de una pradera con tres años de establecida en el Banco de Germoplasma de Forrajes Tropicales, Campo Experimental Rosario Izapa, Instituto Nacional de Investigaciones Forestales Agrícolas y Pecuarias (INIFAP), ubicado en Carretera TapachulaCacahoatán km 18, Tuxtla Chico, Chiapas, México, $14^{\circ} 40^{\prime}$ 16.1' LN, 92 42' 59.1' LO, a 435 msnm. Al momento del corte la planta tenía 180 días de rebrote aproximadamente. El corte se hizo a $20 \mathrm{~cm}$ sobre el nivel del suelo. Se cortaron tallos de aproximadamente $1.5 \mathrm{~cm}$ de largo y se traslaron al terreno donde se estableció el mismo día. El establecimiento del pasto Taiwán en una hectárea, se llevó a cabo en condiciones de temporal en el mes de junio de 2018, previo se preparó el terreno con un barbecho y dos pasos de rastra, después se procedió al establecimiento. El tallo se cortó dejando dos a tres nudos por mata, se colocó en el suelo en forma perpendicular dejando un nudo enterrado a $5 \mathrm{~cm}$, la distancia entre matas fue de aproximadamente 50 $\mathrm{cm}$ y la distancia entre hileras de $80 \mathrm{~cm}$, el control de malezas se hizo de forma manual a 60 días del establecimiento y el corte de uniformidad a 90 días a $30 \mathrm{~cm}$ sobre el nivel del suelo.

\section{Carga animal y manejo del pastoreo}

La fase experimental inició en el mes de junio de 2019, 45 días después del corte de uniformidad se inició el pastoreo con 10 becerros con peso vivo promedio de $252 \mathrm{~kg}$, que se usaron como defoliadores, con una carga de 5.6 U.A. ha ${ }^{-1}$. El sistema de pastoreo fue rotacional en franjas, se asignó diariamente un área de $200 \mathrm{~m}^{2}$ por la mañana, delimitando el área con cerco eléctrico, y los animales se cambiaron de potrero cada $24 \mathrm{~h}$. Los becerros recibieron diariamente $2.5 \mathrm{~kg}$ de un alimento balanceado, se ofreció la mitad por la mañana (7:30 h) y la mitad por la tarde (14:00 h), tuvieron acceso a agua limpia todo el tiempo. En la Tabla 1 se muestran los resultados del análisis químico proximal del alimento balanceado asignado a los becerros en pastoreo.

El experimento se inició al tercer ciclo de pastoreo, con intervalos de 45 días. Se evaluó composición química, variables de crecimiento como altura de planta, largo y ancho de hoja, materia seca acumulada, proporción hoja-tallo y persistencia del pasto Taiwán por el efecto de dos fuentes de fertilización. Los tratamientos se distribuyeron en un diseño de bloques completos al azar, con tres repeticiones (15 plantas por repetición). Los tratamientos fueron: Pasto Taiwán sin fertilización (SF); Pasto Taiwán + fertilizante húmico $(\mathrm{FH}): \mathrm{kg} \mathrm{ha}^{-1}(\mathrm{~N}: 0.770 \mathrm{~kg}$, $\mathrm{P}: 0.490 \mathrm{~kg}, \mathrm{~K}: 1.26 \mathrm{~kg}$, Fuente [P: $\left.\mathrm{P}_{2} \mathrm{O}_{5}, \mathrm{~K}: \mathrm{K}_{2} \mathrm{O}\right]$ ); Pasto Taiwán + fertilizante granulado (FG): $\mathrm{kg} \mathrm{ha}^{-1}$ $\left(\mathrm{N}: 21 \mathrm{~kg}, \mathrm{P}: 17 \mathrm{~kg}, \mathrm{~K}: 3 \mathrm{~kg}\right.$, Fuente [P: $\mathrm{P}_{2} \mathrm{O}_{5}, \mathrm{~K}$ : $\left.\left.\left(\mathrm{K}_{2} \mathrm{O}\right)\right]\right)$.

El fertilizante húmico se diluyó, $10 \mathrm{~mL}$ por litro de agua y se aplicaron $250 \mathrm{~mL}$ por macollo alrededor de la base con una bomba manual los días 1 , 10 y 20 después del pastoreo. Tres días después del pastoreo se aplicó el fertilizante granulado, $50 \mathrm{~g}$ alrededor de la base del macollo y enterrado a $5 \mathrm{~cm}$.

\section{Análisis químico de las muestras}

Las muestras de pasto se obtuvieron de la pradera por triplicado (bloque), según los tratamientos experimentales, consistieron en cinco plantas por bloque, en total 15 plantas. La toma de muestras se realizó a los 45 días de edad de la planta, el corte 


\begin{tabular}{|c|c|c|c|c|}
\hline Determinación & $\begin{array}{c}\text { Alimento } \\
\text { balanceado }\end{array}$ & $\begin{array}{l}\text { Taiwán sin } \\
\text { fertilización }\end{array}$ & $\begin{array}{l}\text { Taiwán + } \\
\text { húmico }\end{array}$ & $\begin{array}{c}\text { Taiwán + } \\
\text { granulado }\end{array}$ \\
\hline Proteína cruda & 19.55 & 7.99 & 9.31 & 11.87 \\
\hline Grasa cruda & 3.89 & 0.96 & 1.02 & 0.72 \\
\hline Fibra cruda & 13.07 & 38.19 & 37.48 & 37.32 \\
\hline Cenizas & 5.53 & 21.33 & 21.11 & 19.31 \\
\hline Fibra detergente neutro & 75.39 & 61.09 & 61.29 & 70.11 \\
\hline Fibra detergente ácido & 16.32 & 43.4 & 44.11 & 50.24 \\
\hline
\end{tabular}

se hizo a $30 \mathrm{~cm}$ del suelo, se picaron a un tamaño de partícula de $1.5 \mathrm{~cm}$, se depositaron aproximadamente $1000 \mathrm{~g}$ en bolsas de papel previamente identificadas, se secaron en horno de aire forzado a $60{ }^{\circ} \mathrm{C}$ por $48 \mathrm{~h}$, se colocaron en bolsas de plástico previamente identificadas y se enviaron al laboratorio para su análisis químico; para el alimento balanceado se tomaron tres muestras de $500 \mathrm{~g}$ de un lote de 500 $\mathrm{kg}$ al momento de elaborarlo, se colocaron en bolsas de plástico identificadas y se enviaron al laboratorio para su análisis. Las determinaciones químicas del pasto Taiwán se realizaron en el Laboratorio de Nutrición Animal del Centro Universitario del Sur, de la Universidad de Guadalajara, ubicado en Ciudad Guzmán, Jalisco, México. Al pasto Taiwán y alimento balanceado se determinó materia seca total (MST), proteína cruda (PC) mediante el método Kjeldahl, extracto etéreo $(E E)$ por el método Soxhlet, fibra cruda (FC) por el método de Weende, cenizas (C) y materia orgánica $(\mathrm{MO})$ por diferencia, todos mediante las técnicas descritas por la AOAC (2012). La determinación de las fracciones de fibra (FDN y FDA) se realizó con alfa amilasa sin la corrección de la ceniza de acuerdo con lo especificado por Van Soest et al. (1991). La composición química del alimento y el pasto Taiwán se muestra en la Tabla 1.

\section{Altura de planta, largo de hoja y ancho de hoja}

La altura de planta (AP), largo de hoja (LH), ancho de hoja $(\mathrm{AH})$, peso seco de planta $(\mathrm{PP})$, peso del tallo $(\mathrm{PT})$, peso de la hoja $(\mathrm{PH})$ y número de hijuelos $(\mathrm{NH})$ se determinó a los 45 días después del pastoreo. La planta se midió desde la base hasta donde dobla la tercera hoja con una cinta métrica de $3.0 \mathrm{~m}$ de largo y $1.0 \mathrm{~mm}$ de precisión; el largo de hoja se determinó desde la inserción del limbo con la vaina hasta el ápice terminal. Ancho de hoja, en la parte media de la hoja con un vernier digital. El número de hijuelos (plantas nuevas) se determinó de forma visual, contabilizando aquellos que emergieron del suelo en la base del macollo. Para determinar el número de macollos vivos de cada bloque, se contabilizó el número de macollos vivos, tomando como macollo vivo aquel que por lo menos tuvo un hijuelo o rebrote. El peso seco total de la planta, se determinó cortando las cepas a $30 \mathrm{~cm}$ sobre el nivel del suelo, el material obtenido se pesó en fresco con una balanza digital con capacidad para $5.0 \mathrm{~kg}$ y precisión de 1.0 g; para peso del tallo y peso de hoja, se separó el tallo de la hoja y se registró el peso fresco de cada parte, luego las muestras se depositaron en bolsas de papel etiquetadas, y se secaron en una estufa de aire forzado a $60{ }^{\circ} \mathrm{C}$ durante $48 \mathrm{~h}$, hasta obtener peso constante y determinar el peso seco. Con estos datos, se obtuvo el porcentaje de materia seca (MS) que se utilizó para obtener la acumulación de materia seca ( $g$ de $M S$ planta $^{-1}$ ). A partir de los pesos seco totales de planta, del tallo y de hoja se calculó el porcentaje de tallo y hoja.

Una segunda evaluación se realizó en el cuarto ciclo de pastoreo en la pradera sin fertilización. Se evaluó el efecto de dos alturas de corte en la persistencia del pasto Taiwán en condiciones de pastoreo intensivo de becerros en engorda, tomando como persistencia el número de hijuelos (nuevas plantas), 45 días después del pastoreo y el número de macollos vivos. Los tratamientos evaluados fueron: pasto Taiwán sin corte de uniformidad después del pastoreo (SC); pasto Taiwán con corte de uniformidad a $30 \mathrm{~cm}$ de altura después del pastoreo (C30); 
y pasto Taiwán con corte de uniformidad a $50 \mathrm{~cm}$ de altura después del pastoreo (C50). El corte de uniformidad se hizo al día siguiente de haber salido los becerros del pastoreo, la altura del forraje residual fue de aproximadamente $80 \mathrm{~cm}$.

\section{Variables evaluadas}

Número de hijuelos, aquellos que ya habían emergido del suelo en la base del macollo 45 días después del pastoreo o corte de uniformidad. Número de macollos vivos 45 días después del pastoreo, para ello de cada bloque que estuvo formado por 25 macollos se contabilizó el número de macollos vivos, tomando como macollo vivo aquel que por lo menos tuvo un hijuelo o rebrote.

\section{Diseño experimental y análisis estadístico}

Se utilizó un diseño experimental bloques completos al azar con tres repeticiones y 15 plantas por repetición. Los datos se sometieron a un análisis de varianza para determinar las diferencias estadísticas entre los tratamientos. La comparación de medias se hizo con la prueba de Tukey con un alpha $=0.05$, con el paquete estadístico SAS, Ver. 9.1.3 (SAS 2006).

\section{RESULTADOS}

Se observó que el contenido de proteína cruda del pasto Taiwán, aumentó de 7.99 a 9.31\% (1.32 unidades) cuando se aplicó fertilizante húmico, y a 11.87\% (3.88 unidades) cuando se aplicó fertilizante granulado. En el pasto que recibió la fertilización granulada, el contenido de FDN tuvo un aumento de $9.02 \%$, y el contenido de FDA de $6.34 \%$ con respecto al pasto que no recibió fertilización (Tabla 1).

En la Tabla 2 se muestran los resultados de crecimiento a los 45 días con dos fuentes de fertilización. No se observaron diferencias entre tratamientos en altura de planta y ancho de hoja ( $P$ $>0.05$ ), mientras que el largo de hoja fue menor en el pasto Taiwán con aplicación del fertilizante húmico $(P<0.05)$. Para el peso seco de planta, de tallo y de hoja a 45 días de edad de corte, no hubo diferencia entre tratamientos $(P>0.05)$, ni para el porcentaje de hoja y tallo. Pero el pasto donde se aplicó fertilizante granulado tuvo $55.26 \%$ de hoja comparado con $48.88 \%$ en donde no se aplicó fertilizante, lo que indica una diferencia del $6.46 \%$. En relación con el número de hijuelos y porcentaje de macollos vivos (Tabla 3), los resultados muestran que no hubo diferencia entre tratamientos por efecto de la fuente de fertilización $(P>0.05)$; mientras que el corte de uniformidad a 30 y $50 \mathrm{~cm}$ tuvo efecto significativo en el número de hijuelos $(P<0.01)$.

\section{DISCUSIÓN}

El contenido de PC del alimento balanceado fue del $19.55 \%$ (Tabla 1), el cual fue diseñado para complementar el aporte de PC del pasto Taiwán y cubrir el requerimiento diario de proteína de becerros de $250 \mathrm{~kg}$ de peso vivo (NRC 2000). Los valores de PC del pasto Taiwán observados (Tabla 1), muestran que cuando se aplicó fertilizante húmico, aumentó de 7.99 a 9.31\%, y cuando se aplicó fertilizante granulado a $11.87 \%$. De acuerdo con los resultados obtenidos con la aplicación de fertilizante granulado se tuvo un incremento en el contenido de PC del $3.88 \%$ en comparación con el tratamiento donde no se aplicó fertilizante.

De acuerdo con los resultados de crecimiento de la planta (Tabla 2), no hubo efecto por la fuente de fertilización en altura de planta, ancho de hoja, peso seco del tallo, peso seco de la hoja, porcentaje de tallo y porcentaje de hoja $(P>0.05)$, lo que indica que la fuente de fertilización no modificó la estructura de la planta ni la acumulación de materia seca, pero aumentó el contenido de proteína en 1.32 y 3.88\% cuando se aplicó fertilizante húmico y granulado de manera respectiva. Este incremento puede estar relacionado con la aplicación de K, la cantidad en cada tratamiento fue de $1.26 \mathrm{~kg}$ de $\mathrm{K} \mathrm{ha}^{-1}$ en la aplicación de fertilizante húmico y de $3.00 \mathrm{~kg} \mathrm{ha}^{-1}$ en la aplicación de fertilizante granulado en el ciclo de corte de 45 días. Al respecto, se sabe que la función de este nutriente es como catalizador en las reacciones enzimáticas responsables de la síntesis de proteína, activa las enzimas involucradas en la fotosíntesis, el metabolismo de las proteínas y de carbohidratos. Ya 
Tabla 2. Crecimiento, materia seca acumulada y proporción hoja-tallo del pasto Pennisetum purpureum cv. Taiwán con aplicación de fertilizante húmico o granulado en pastoreo intensivo de becerros en engorda.

\begin{tabular}{lcccc}
\hline Variables & $\begin{array}{c}\text { Taiwán sin } \\
\text { fertilización }\end{array}$ & $\begin{array}{c}\text { Taiwán + } \\
\text { húmico }\end{array}$ & $\begin{array}{c}\text { Taiwán }+ \\
\text { granulado }\end{array}$ & EEM \\
\hline \multicolumn{5}{c}{ Crecimiento de la planta } \\
Altura de planta (cm) & $164.2^{a}$ & $160.8^{a}$ & $166.8^{a}$ & 15.9 \\
Largo de hoja (cm) & $106.3^{a}$ & $100.8^{b}$ & $108.1^{a}$ & 12.3 \\
Ancho de hoja (cm) & $3.34^{a}$ & $3.9^{a}$ & $3.24^{a}$ & 0.60 \\
\multicolumn{5}{c}{ Materia seca acumulada } \\
Peso seco de planta (g) & $46.0^{a}$ & $52.7^{a}$ & $50.7^{a}$ & 14.6 \\
Peso seco de tallo (g) & $23.4^{a}$ & $26.6^{a}$ & $22.1^{a}$ & 9.7 \\
Peso seco de hoja (g) & $22.6^{a}$ & $26.1^{a}$ & $28.1^{a}$ & 11.8 \\
\multicolumn{5}{c}{ Proporción de tallo:hoja (\%) $^{a}$} \\
Porcentaje de tallo & $51.1^{a}$ & $50.8^{a}$ & $44.7^{a}$ & 12.9 \\
Porcentaje de hoja & $48.9^{a}$ & $49.2^{a}$ & $55.3^{a}$ & 12.9 \\
\hline EEM: error estándar de la media; ${ }^{a, b}$ valores promedio con literales diferentes \\
en la misma fila, son estadísticamente diferentes (Tukey; p<0.05).
\end{tabular}

Tabla 3. Número de hijuelos y macollos vivos del pasto Pennisetum purpureum cv. Taiwán con dos fuentes de fertilización y dos alturas de corte de uniformidad.

\begin{tabular}{lcccc}
\hline Variable & \multicolumn{4}{c}{ Fuentes de fertilización } \\
\hline & \multicolumn{1}{c}{ Taiwán sin } & Taiwán + & Taiwán + & EEM \\
& fertilización & húmico & granulado & \\
\hline Número de hijuelos & $11.6^{a}$ & $10.2^{a}$ & $10.7^{a}$ & 4.6 \\
Porcentaje de macollos vivos & $100.0^{a}$ & $97.3^{a}$ & $97.3^{a}$ & 5.2 \\
\cline { 2 - 5 } & \multicolumn{4}{c}{ Alturas de corte de uniformidad } \\
& Taiwán sin & Taiwán + corte & Taiwán + & EEM \\
\cline { 2 - 5 } Número de hijuelos & corte & a $30 \mathrm{~cm}$ & corte a $50 \mathrm{~cm}$ & \\
\cline { 2 - 5 } Número de macollos vivos & $7.3^{b}$ & $15.4^{a}$ & $17.8^{a}$ & 6.5 \\
\hline EEM: error estándar de la media; ${ }^{a, b}$ valores promedio con literales diferentes en la misma
\end{tabular}

EEM: error estándar de la media; ${ }^{a, b}$ valores promedio con literales diferentes en la misma fila, son estadísticamente diferentes (Tukey; $p<0.05$ ).

que la enzima nitrato reductasa cataliza la reacción para la síntesis de proteínas y el $\mathrm{K}$ es el responsable de la activación enzimática (Patil 2011).

En relación con el efecto de la fertilización con $\mathrm{N}$, su función está relacionada con la síntesis de clorofila, es un elemento esencial en la fotosíntesis, acelera la división celular para la formación de nuevos tejidos, con ello aumenta la biomasa radicular y aérea de la planta (Bélanger y Gastal 2000). Por ello, el efecto principal de la fertilización con $\mathrm{N}$ en los pastos, es el aumento en el rendimiento de la materia seca (Rojas et al. 2017), y tiene un efecto limitado en el contenido de PC. Lo que puede explicar la respuesta limitada en el contenido de PC del tratamiento con la fertilización con N. Al respecto, Roncallo et al. (2012) indican que no hubo efecto de la fertilización nitrogenada $\left(0 \% \mathrm{~N}, 50 \mathrm{~kg} \mathrm{ha}^{-1} \mathrm{~N}\right.$, Azotobacter, $50 \mathrm{~kg} \mathrm{ha}^{-1}$ de estiércol vacuno seco, y Leucaena) en el contenido de PC de los pastos Elefante (Pennisetum pur- pureum), Taiwán (Pennisetum purpureum x Pennisetum typhoides) y Maralfalfa (Pennisetum $\mathrm{sp}$ ) cosechados a 50 días de edad $(5.14,5.47,6.02,5.37$ y $5.75 \%)$ de manera respectiva para las fuentes de fertilización mencionadas. En otro estudio, Márquez et al. (2007) observaron que el contenido de PC del pasto Taiwán fue de 7.62 y $8.08 \%$ cuando la aplicación de $\mathrm{N}$ en forma de urea fue de 343 y $686 \mathrm{~kg} \mathrm{ha}^{-1}$ año ${ }^{-1}$ de manera respectiva, sin efecto significativo.

La dosis de fertilización es importante para que haya efecto positivo en el rendimiento de materia seca, en el presente estudio no hubo efecto significativo en peso seco de la planta $(P>0.05)$, posiblemente por la dosis de $\mathrm{N}$ aplicada que fue de 0.770 $\mathrm{kg} \mathrm{ha}^{-1}$ de la fuente húmica y $21 \mathrm{~kg} \mathrm{ha}^{-1}$ de la granulada en un ciclo de pastoreo de 45 días. Al respecto, López et al. (2020), evaluaron la eficiencia en la extracción de N, P y K cuando se aplicó fertilización al pasto Pennisetum cv. Maralfalfa en do- 
sis de 141-43-20 (N-P-K), observando que la eficiencia fue de 48, 39 y $104 \%$, respectivamente. Los resultados obtenidos en peso seco de planta (Tabla 2) son menores a lo reportado por Madera et al. (2013), quienes mencionan que en el pasto Pennisetum purpureum cv. Morado, la producción de MS por planta a 45 días de edad fue de $115.59 \mathrm{~g}$.

El contenido de FDN obtenido, muestra un aumento del $9.02 \%$ en el pasto Taiwán que recibió el fertilizante granulado, en relación con el pasto no fertilizado, lo que indica que se tuvo mayor acumulación de paredes celulares por efecto del fertilizante granulado. Sobre lo mismo Roncallo et al. (2012), no observaron diferencia en FDN y FDA cuando aplicaron cinco fuentes de fertilización nitrogenada al pasto Taiwán, obteniendo valores de FDN y FDA de 70.2 y $44.70 \%$ respectivamente al aplicar $0.0 \%$ y $50 \mathrm{~kg}$ de $\mathrm{N}$ ha ${ }^{-1}$. También González et al. (2011) obtuvieron valores de FDN de 54.5\%, Correa et al. (2006) de $37.0 \%$ de FDA en pasto Maralfalfa sin aplicación de fertilizante.

No se observó diferencia en altura de planta y ancho de hoja $(P<0.05)$ por efecto de la fuente de fertilización (Tabla 2). Al respecto se sabe que la cantidad y calidad del forraje producido por los pastos está determinada por la morfología de la planta (Calzada et al. 2014). Pero aun así los resultados obtenidos, son mayores a lo reportado por Madera et al. (2013), quienes registraron altura de la planta de Pennisetum purpureum cv Morado a 45 días de rebrote de 74.75 $\mathrm{cm}$, largo de hoja de $60.0 \mathrm{~cm}$ y ancho de hoja de 2.63 $\mathrm{cm}$, y también a los valores reportados por Vívas et al. (2019) de $150 \mathrm{~cm}$ de altura de planta a 50 días de edad. Mientras mayor es la altura de la planta, mayor es la proporción de hojas sombreadas y se tiene correlación negativa con la biomasa foliar, de allí la importancia de cosechar el forraje en el momento óptimo, antes que inicie la muerte de hojas (Anten et al. 1999). Con respecto al porcentaje de hoja y tallo, los resultados son mayores a lo observado por Ramos et al. (2015), quienes reportan que el porcentaje de hoja en el pasto Pennisetum purpureum cv. Taiwán a 90 días de edad con la aplicación de $200 \mathrm{~kg}$ de $\mathrm{N}$ y $60 \mathrm{~kg}$ de $\mathrm{P}$ ha ${ }^{-1}$ fue de $38 \%$; mientras que Goyes et al. (2018), observaron que la relación hoja:tallo de los cultivares King grass, Camerún, Maralfalfa y Elefante enano, con fertilización de $212 \mathrm{~kg} \mathrm{~N} \mathrm{ha}^{-1}, 39.2 \mathrm{~kg} \mathrm{P}$ $\mathrm{ha}^{-1}$ y $36.2 \mathrm{~kg} \mathrm{~K} \mathrm{ha}^{-1}$ fue de $1.28,1.1,0.82$ y 0.66 , respectivamente.

Para el efecto de la fuente de fertilización y la altura de corte en el número de hijuelos, la fuente de fertilización no tuvo efecto significativo $(P>0.05)$. Mientras que otros estudios reportan que el rebrote de nuevos tallos es estimulado por la aplicación con $\mathrm{N}$ (Mazzanti et al. 1994); pero el efecto de la fertilización con $\mathrm{N}$ en el desarrollo de hijuelos es limitada por factores como la densidad de macollos e índice de área foliar y por el efecto de sombra que ejercen en la base del macollo (Simon y Lemaire 1987), por tanto, es importante que después del pastoreo o corte, el área foliar remanente permita buena luminosidad en la base del macollo. De acuerdo con lo anterior, en este estudio, al evaluar dos alturas de corte de uniformidad después del pastoreo (Tabla 3 ), el número de hijuelos fue mayor cuando se hizo corte de uniformidad a $30 \mathrm{~cm}$ y a $50 \mathrm{~cm}(P<0.01)$, comparado con el pasto donde no se realizó corte de uniformidad después del pastoreo, lo que indica que el corte de uniformidad después del pastoreo es un manejo que puede estimular el crecimiento de nuevas plantas. El mayor número de hijuelos observado cuando se dio corte de uniformidad a 30 y $50 \mathrm{~cm}$, pudo deberse a la mayor incidencia de luz en la base del macollo, al activar las yemas axilares remanentes que favorece la aparición y crecimiento de nuevos tallos (Deregibus et al. 1983, Casal et al. 1985); así mismo, el grado de defoliación determina la regeneración del área foliar y define la cantidad de yemas que potencialmente pueden desarrollar macollos, componentes principales del crecimiento y la persistencia de los pastos (Skinner y Nelson 1995, Rojas et al. 2017). Por ello es recomendable uniformar la pradera cuando el crecimiento de las hojas disminuye (Calzada et al. 2014), debido a que la etapa morfológica y fisiológica en la que se encuentra la planta al momento del corte determina la persistencia y el vigor de los rebrotes (Pérez et al. 2004). Mientras que Rodolfo et al. (2015), no observaron diferencia en el número de tallos nuevos, al evaluar dos niveles de defoliación (50 y 70\%) en el pasto Elefante (Pen- 
nisetum purpureum).

\section{CONCLUSIONES}

La aplicación de fertilizante húmico o fertilizante granulado al pasto Pennisetum purpureum cv. Taiwán, no afectó las variables de crecimiento, producción de materia seca de la planta, porcentaje de hoja y tallo, número de hijuelos y porcentaje de macollos vivos; mientras que el contenido de proteína cruda aumentó $3.88 \%$ cuando se aplicó el fertilizante granulado. El corte de uniformidad a $30 \mathrm{y}$ $50 \mathrm{~cm}$ después del pastoreo estimuló el desarrollo de nuevos hijuelos, lo que promueve la persistencia de la pradera en condiciones de pastoreo intensivo de becerros en engorda.

\section{LITERATURA CITADA}

AOAC (2012) Official Methods of Analysis of the Association of Official Analytical. $19^{\text {th }}$ Edition. Association of Official Analytical Chemists. Official Methods of Analysis Gaithersburg, Mary Land, USA. 44p.

Anten NPR, Hirose T (1999) Interspecific differences in above ground growth patterns result in spatial and temporal partitioning of light among species in a tall-grass meadow Journal Ecology 87: 583-597.

Arboleda D, Tombe A, Morales VS (2013) Propuesta para el establecimiento de especies arbóreas y arbustivas con potencial forrajero en sistemas de producción ganadera del trópico alto colombiano. Biotecnología en el Sector Agropecuario y Agroindustrial 11:154-163.

Araya MM, Boschinni FC (2005) Producción de forraje y calidad nutricional de variedades de Pennisetum purpureum en la meseta central de Costa Rica. Agronomía Mesoamericana 16: 37-43.

Bélanger G, Gastal F (2000) Nitrogen utilization by forage grasses. Canadian Journal of Plant Science 80: 11-20.

Calzada MJM, Enriquez QJF, Hernández GA, Ortega JE, Mendoza PSI (2014) Análisis de crecimiento del pasto Maralfalfa (Pennisetum sp.) en clima cálido subhúmedo. Revista Mexicana de Ciencias Pecuarias 5: 247260.

Casal JJ, Deregibus VA, Sánchez RA (1985) Variation in tiller dynamics and morphology in Lolium multiflorum Lam. Vegetative and reproductive plants as affected by differences in red/far-red irradiation. Annals of Botany 56: 553-559.

Correa H (2006) Calidad nutricional del pasto Maralfalfa (Pennisetum sp) cosechado a dos edades de rebrote (en línea). Livestock Research for Rural Development 18: 326-335.

Deregibus VA, Sánchez RA and Casal JJ (1983) Effects of light quality on tiller production in Lolium spp. Plant Physiology 72: 900-912.

García E (2004) Modificaciones al Sistema de Clasificación Climática de Köppen (adaptado a las condiciones de la República Mexicana. $5^{\mathrm{a}}$ edición. Instituto de Geografía, UNAM. México. 91p.

González I, Betancourt M, Fuenmayor A, Lugo M (2011) Producción y composición química de forraje de dos especies de pasto Elefante (Pennisetum sp.) en el Noroccidental de Venezuela. Zootecnia Tropical 29: 103-112.

Goyes VFR, Martínez GJC, Soquicela RRA, Catota GLD, Acosta JMV, Barros BFB (2018) Fertilización y producción del pasto del género Pennisetum en Santo Domingo, Ecuador. Tropical and Subtropical Agroecosystems 21: 213-223.

Hinojosa YLA, Yépez ND, Rodal CF, Ríos OA, Claros BR, Suarez NT, Jiménez LE (2014) Producción y características agronómicas de cuatro variedades de pasto de corte del género Pennisetum en Trinidad Bolivia Agrociencias Amazonia 3: 28-35. 
INEGI. Instituto Nacional de Estadística y Geografía (2017) Anuario estadístico y geográfico de Chiapas https:// www.inegi.org.mx/contenido/productos/prod_serv/contenidos/espanol/bvinegi/productos/nueva_estruc/anua rios_2017/702825094836.pdf. Fecha de consulta: 5 de Febrero de 2021.

López GI, Enriquez QJF (2011) Paquete tecnológico zacate Pennisetum purpupureum: Establecimiento y producción. Programa Estratégico para el Desarrollo Rural Sustentable de la Región Sur Sureste de México: Trópico húmedo. CIRGOC. Campo Experimental "La Posta", INIFAP, Veracruz. 7p. https://docplayer.es/4068 6695-Programa-estrategico-para-el-desarrollo-rural-sustentable-de-la-region-sur-sureste-de-mexico-tropicohumedo-2011.html. Fecha de consulta: 5 de Febrero de 2021.

López AO, Vinay VJC, Villegas AY, López GI, Lozano TS (2020) Dinámica de crecimiento y curvas de extracción de nutrientes de Pennisetum sp. (Maralfalfa). Revista Mexicana de Ciencias Pecuarias 11: 255-265.

Madera NB, Ortiz B, Bacab HM, Magaña H (2013) Influencia de la edad de corte del pasto morado (Pennisetum purpureum) en la producción y digestibilidad in vitro de la materia seca. Avances en Investigación Agropecuaria 17: 41-52.

Márquez F, Sánchez T, Urbano D, Dávila C (2007) Evaluación de la frecuencia de corte y tipos de fertilización sobre tres genotipos de pasto elefante (Pennisetum purpureum). 1. Rendimiento y contenido de proteína. Zootecnia Tropical 25: 253-259.

May EFV, Rubio JGR, Ruelas AFC, Aguilar MHY, Cabrera-Torres EJ (2011) Mineral content of forage species found in sheep farms in the state of Yucatán, México. Revista Mexicana de Ciencias Pecuarias 2: 465-475.

Mazzanti A, Lemaire G, Gastel F (1994) The effect of nitrogen fertilization upon the herbage Production of tall fescue swards continuously grazed with sheep. I. Herbage growth dynamics. Grass and Forage Science 49: 111-120.

Molina RM, Olea PR, Galindo MFA, Arroiga JCM (2019) Life cycle assessment of three tropical livestock systems in Campeche, Mexico: case of study. Tropical and Subtropical Agroecosystems 22:127-141.

Nava JJ, Gutiérrez E, Herrera RS, Zavala F, Olivares E, Treviño JE, Bernal H, Valdés CGS (2013) Rendimiento y composición química del pasto CT-115 (Pennisetum purpureum) establecido a dos densidades y en dos fechas de siembra en Marín, Nuevo León, México. Revista Cubana de Ciencia Agrícola 47: 419-424.

NRC (2000) Nutrient requirements of beef cattle. Seventh Revised Edition. National Research Council. The National Academies Press. Washington, D. C. USA. 248p.

Patil RB (2011) Role of potassium humate on growth and yield of soybean and black gram. International Journal of Pharma and Bio Sciences 2: 242-246.

Perdomo CMF, Peña BLF, Carvajal YJD, Murillo LSLY (2017) Relación nutrición-fertilidad en hembras bovinas en clima tropical. Revista Electrónica de Veterinaria 18:1-19.

Pérez AJA, García ME, Enríquez QJF, Quero CAR, Pérez JJ, Hernández GA (2004) Análisis de crecimiento, área foliar específica y concentración de nitrógeno en hojas de pasto "Mulato" (Brachiaria hibrido cv.). Técnica Pecuaria México 42: 447-458.

Quero CAR, Miranda JL (2013) Gramíneas forrajeras nativas de México. Recolecta y aprovechamiento sistemático. In: Quero-Carrillo AR (ed.) Gramíneas introducidas: Importancia e impacto en ecosistemas ganaderos. Colegio de Postgraduados Montecillo, Texcoco, Estado de México. pp: 1-23.

Ramírez RO, Hernández GA, Carneiro da Silva S, Pérez PJ, Jacauna de Souza JS, Castro RR, Enríquez JF (2010) Características morfogenéticas y su influencia en el rendimiento del pasto mombasa, cosechado a diferentes edades de corte. Tropical and Subtropical Agroecosystems. 12: 303-311. 
Ramos TS, Victoria GCA, Sandoval GJJ (2015) Temporada, fertilización y rendimiento de variedad de Pennisetum purpureum. Agrociencia 49: 837-844.

Rodolfo GR, Schmitt D, Días MK, Sbrissia AF (2015) Levels of defoliation and Growth dynamics in elephant grass swards. Ciencia Rural. Santa María 45: 1299-1304.

Rojas GAR, ventura RJ, Hernández GA, Joaquín CS, Maldonado PMA, Reyes VI (2017) Dinámica poblacional de tallos de ovillo (Dactylis glomerata L.) solo y asociado con ballico perenne (Lolium perenne L.) y trébol blando (Trifolium repens L.). Revista Mexicana de Ciencias Pecuarias 8: 419-428.

Roncallo BF, Sierra AAM, Castro ER (2012) Rendimiento de forraje de gramíneas de corte y efecto sobre la calidad composicional y producción de leche en el Caribe seco. Revista Corpoica-Ciencia y Tecnología Agropecuaria 13: 71-78.

SIAP (2019) Población Ganadera. Inventario bovino para carne; Inventario bovino para leche. México. https:// www.gob.mx/siap/documentos/poblacion-ganadera-136762. Fecha de consulta: 5 de febrero 2021.

Simon JC, Lemaire G (1987) Tillering and leaf area index in grass in vegetative phase. Grass and Forage Science 42: 373-380.

Skinner RH, Nelson CJ (1995) Elongation of the grass leaf and its relationship to the phyllochron. Crop Science 35: 4-10.

SAS (2006) User's guide. Version 9.1.3. Statistical Analysis System Institute Inc. Cary, NC, USA. 443p.

Valentine I, Mathew C (1999) Plant development and yield. In: White J, Hodgson J (ed). NZ pasture crop science auckland NZ. Oxford University Press. USA. pp: 11-27.

Van Soest PJ, Robertson B, Lewis BA (1991) Methods for dietary fiber, neutral detergent fiber and nonstarch polysaccharides in relation to animal nutrition. Symposium: carbohydrate methodology, metabolism, and nutritional implications in dairy cattle. Journal of Dairy Science 74: 3583-3597.

Vivas QNJ, Criollo DMZ, Cedeño GMC (2019) Frecuencia de corte del pasto Elefante morado Pennisetum purpureum Schumach. Biotecnología en el Sector Agropecuario 17: 41-55. 